\title{
EVALUACIÓN DE PROGRAMAS DE SALUD ELECTRÓNICA EN EL PERÚ: ENFOQUE MULTIDISCIPLINARIO Y PERSPECTIVAS ACTUALES
}

\author{
Milagro Henríquez-Suarez ${ }^{1,2, a}$, Charito E. Becerra-Vera ${ }^{1, b}$, Elena L. Laos-Fernández ${ }^{1,2, c}$, \\ Elizabeth Espinoza-Portilla ${ }^{3, d}$
}

\begin{abstract}
RESUMEN
La salud electrónica (eSalud) requiere un enfoque multidisciplinario que incluya diversos campos como la medicina, la informática, ingenierías e, incluso, las ciencias sociales como la sociología, la antropología, el trabajo social, la administración, el derecho y las ciencias de la comunicación. Al evaluar la eSalud debemos tener en cuenta que las tecnologías de información y comunicación (TIC) son solo una parte del sistema de información de una organización; es fundamental considerar los factores humanos involucrados en los procesos del sistema de salud, y tomar en cuenta el entorno y el ecosistema en el que se utilizan las TIC en salud. El objetivo de este artículo es describir la situación actual de la evaluación de experiencias de eSalud en el Perú y discutir las estrategias de evaluación multidisciplinaria que deben considerarse para lograr éxito y sostenibilidad de estas iniciativas, basadas en la experiencia nacional e internacional.
\end{abstract}

Palabras clave: eSalud, Telemedicina, Evaluación, Estudios de evaluación (fuente: DeCS BIREME)

\section{EVALUATION OF ELECTRONIC HEALTH PROGRAMS IN PERU: MULTIDISCIPLINARY APPROACH AND CURRENT PERSPECTIVES}

\begin{abstract}
Electronic health (eHealth) requires a multidisciplinary approach and involves different areas of knowledge, including medicine, computer science, engineering, sociology, anthropology, social work, administration, law, and communication sciences. The assessment of eHealth should consider that information and communication technologies (ICTs) are only part of the information system of an organization. Understanding the human factors involved in health systems, the environment, and the contexts in which ICTs are used in health is essential. The objectives of this study were to describe the status of the assessment of eHealth in Peru and to discuss the strategies for multidisciplinary evaluation that should be considered to achieve the success and sustainability of these initiatives based on national and international experience.
\end{abstract}

Key words: eHealth, Telemedicine, Evaluation, Evaluation studies (source: MeSH NLM).

\section{INTRODUCCIÓN}

La Organización Mundial de la Salud (OMS) define la salud electrónica (eSalud) como: El apoyo que la utilización costo-eficaz y segura de las tecnologías de la información y las comunicaciones ofrece a la salud y a los ámbitos relacionados con ella, con inclusión de los servicios de atención de salud, la vigilancia y la documentación sanitaria, así como la educación, los conocimientos y las investigaciones en materia de salud ${ }^{(1)}$. Este concepto se resalta en la Estrategia y Plan de Acción Sobre eSalud
(2012 - 2017) de la Organización Panamericana de la Salud (OPS), que busca: Mejorar el acceso a los servicios de salud y su calidad, gracias a la utilización de las tecnologías de la información y de las comunicaciones (TIC). El documento menciona como componentes de la eSalud a las historias clínicas electrónicas, la telesalud o telemedicina, la mSalud (salud móvil), la capacitación a través de Internet y la estandarización e interoperabilidad ${ }^{(2)}$.

En Latinoamérica se han desarrollado múltiples iniciativas basadas en eSalud, y Perú no ha sido la excepción, existen

\footnotetext{
Facultad de Ciencias Sociales, Universidad Nacional José Faustino Sánchez Carrión. Huacho, Perú.

Escuela de Posgrado, Universidad Nacional José Faustino Sánchez Carrión. Huacho, Perú.

Instituto de Gobierno y de Gestión Pública, Universidad de San Martín de Porres. Lima, Perú.

Licenciada en Trabajo Social, magíster en Salud Pública y doctora en Administración; ${ }^{\mathrm{b}}$ licenciada en Trabajo Social, magíster en Investigación y Docencia Universitaria y doctora en Ciencias de la Educación; ' licenciada en Trabajo Social, magíster en Docencia Superior e Investigación Universitaria y doctora en Ciencias de la Educación; ${ }^{\mathrm{A}}$ médico cirujano, magíster en Estudios de Políticas Públicas, magíster en Demografía y Población y doctora en Gobierno y Políticas Públicas.

Recibido: 27/06/2017 Aprobado: 20/09/2017 En línea: 13/11/2017
}

Citar como: Henríquez-Suarez M, Becerra-Vera CE, Laos-Fernández EL, Espinoza-Portilla E. Evaluación de programas de salud electrónica en el Perú: enfoque multidisciplinario y perspectivas actuales. Rev Peru Med Exp Salud Publica. 2017;34(4):731-7. doi:10.17843/rpmesp.2017.344.2974 
en la actualidad diversos proyectos que involucran una serie de tecnologías, como el uso de dispositivos móviles, Internet y las redes sociales. En el 2015, Gozzer reportó más de 30 experiencias de telesalud en el Perú y resaltó la necesidad de un trabajo colaborativo y la importancia del financiamiento para el escalamiento a nivel nacional de intervenciones innovadoras con un enfoque sistémico, con el fin de potenciar el impacto en las políticas de salud. Es predecible el gran interés por el uso de las TIC en intervenciones sanitarias, ya que se ha visto un crecimiento del mismo, tanto en áreas rurales como urbanas del Perú (3).

Para asegurar la eficiencia de estos programas, es necesario monitorear de forma continua las futuras iniciativas de eSalud. Asimismo, esta requiere un enfoque multidisciplinario que incluya diversos campos como la Medicina, la Informática, la Ingeniería e, incluso, las ciencias sociales: la Sociología, la Antropología, el Trabajo Social, la Administración, el Derecho y las Ciencias de la Comunicación. El objetivo de este artículo es describir la situación actual de la evaluación de experiencias de eSalud en Perú y discutir las estrategias de evaluación multidisciplinaria que deben considerarse para lograr éxito y sostenibilidad de estas iniciativas, basadas en la experiencia nacional e internacional.

\section{SITUACIÓN ACTUAL DE LA EVALUACIÓN DE LAS EXPERIENCIAS DE SALUD ELECTRÓNICA EN EL PERÚ}

En el Perú, Gozzer reportó 38 experiencias en telesalud, aunque no todas se encontraban en actividad al momento del reporte ${ }^{(3)}$. Para el presente artículo se realizó una búsqueda bibliográfica de artículos con enfoque cuantitativo, utilizando la base de datos Scopus (https://www.scopus.com/) y el buscador Google Académico (https://scholar.google.com. pe/) para conocer si los proyectos, ya implementados, fueron evaluados a la fecha $\mathrm{o}$, al menos, consideraron un plan de evaluación en su estructura. Adicionalmente, se realizó una búsqueda en Google (https://www.google.com. pe/) y también se procedió a una revisión manual de las referencias de los artículos seleccionados. Se incluyeron artículos publicados entre los años 2000 al 2015, en inglés o en español, pero con al menos el resumen en inglés. No se incluyeron artículos de revisión, artículos publicados en "sección especial", artículos de simposio, artículos de "opinión y análisis", artículos de factibilidad, capítulos de libros, comunicaciones cortas ni cartas al editor.

La estrategia de búsqueda en Scopus fue:

TITLE-ABS-KEY (telemedicine OR ehealth OR telehealth OR mhealth) OR TITLE-ABS (cell AND phones) OR TITLE-ABS (cellular AND phone) OR TITLE-
ABS (text AND messaging) AND TITLE-ABS (peru) AND ALL (evaluation) AND (EXCLUDE (DOCTYPE, "re" )) AND (EXCLUDE (DOCTYPE, "ch") ) AND (EXCLUDE (DOCTYPE, "cr") ) AND (EXCLUDE (SRCTYPE, "p" ))

Las palabras clave de búsqueda en Google Académico y en Google, incluyeron:

telemedicine OR Ehealth OR telehealth OR (mhealth OR SMS OR cell phone) AND peru AND evaluation

Para los tipos de evaluación, se utilizó la siguiente clasificación basada en experiencias previas ${ }^{(4)}$ de evaluación de programas de eSalud:

a. Historias clínicas electrónicas. Un registro digital de información relacionada a la salud sobre un individuo, que puede ser creada, administrada o consultada por clínicos o por el personal de salud.

b. Sistema de información de laboratorio. Un sistema para actividades específicas del laboratorio o para reportar resultados a administradores y al personal de salud.

c. Sistema de información de farmacia. Cualquier sistema usado para ordenar, entregar o rastrear medicamentos $\mathrm{u}$ órdenes de medicamentos, incluyendo sistemas de entrada computarizados.

d. Sistema de registro de pacientes o de citas. Cualquier sistema usado para monitorizar y administrar el movimiento de los pacientes.

e. Sistema de monitoreo, seguimiento y evaluación de pacientes. Cualquier sistema usado para el reporte de información, monitoreo de iniciativas y programas relacionados con el seguimiento y evaluación del estado del paciente.

f. Sistema de apoyo para las decisiones clínicas. Un sistema diseñado para mejorar la toma de decisiones clínicas, en el cual las características individuales de los pacientes son alineadas con una base computarizada de conocimiento y algoritmos en el software para generar recomendaciones específicas para el paciente.

g. Sistema de recordatorios para el paciente. Un sistema usado para estimular a los pacientes para realizar una acción específica, por ejemplo, tomar medicamentos o acudir al centro de salud.

h. Sistema de recolección de datos / investigación. Cualquier sistema usado para recolectar información desde diferentes lugares o para guardar, administrar o reportar información usada para fines de investigación.

Se recuperaron 18 publicaciones, cinco por Scopus, 10 por Google Académico, uno por Google y dos por revisión manual. Los resultados de la búsqueda se resumen en la Tabla 1.

Existen otras áreas que han sido abordadas en estudios peruanos que han evaluado cursos relacionados a 
Tabla 1. Experiencias de evaluación de intervenciones en salud electrónica en Perú

\begin{tabular}{|c|c|c|}
\hline $\begin{array}{l}\text { Aplicativo/sistema de } \\
\text { información (institución) }\end{array}$ & Categoría & Tipo de evaluación \\
\hline $\begin{array}{l}\text { NETLAB (Instituto } \\
\text { Nacional de Salud) }{ }^{(5)}\end{array}$ & $\begin{array}{c}\text { Sistema de información } \\
\text { de laboratorio }\end{array}$ & Antes y después \\
\hline $\begin{array}{l}\text { Historias clínicas } \\
\text { electrónicas (Programa } \\
\text { Nacional de Tuberculosis } \\
\text { (PNT), Socios en Salud) }{ }^{(6)}\end{array}$ & $\begin{array}{c}\text { Sistema de información } \\
\text { de laboratorio }\end{array}$ & $\begin{array}{l}\text { Modelo actual versus } \\
\text { intervención }\end{array}$ \\
\hline $\begin{array}{l}\text { Historias clínicas } \\
\text { electrónicas (PNT, Socios } \\
\text { en Salud) }{ }^{(7)}\end{array}$ & $\begin{array}{c}\text { Sistemas de } \\
\text { información de } \\
\text { laboratorio }\end{array}$ & $\begin{array}{l}\text { Modelo propuesto } \\
\text { versus uso actual }\end{array}$ \\
\hline $\begin{array}{l}\text { Historias clínicas } \\
\text { electrónicas (PNT, Socios } \\
\text { en Salud) }{ }^{(8)}\end{array}$ & $\begin{array}{l}\text { Sistemas de } \\
\text { información de } \\
\text { laboratorio }\end{array}$ & $\begin{array}{l}\text { Ensayo controlado } \\
\text { externamente tipo } \\
\text { antes y después }\end{array}$ \\
\hline $\begin{array}{l}\text { eChasqui (Instituto } \\
\text { Nacional de Salud) }^{(9)}\end{array}$ & $\begin{array}{l}\text { Sistemas de } \\
\text { información de } \\
\text { laboratorio }\end{array}$ & $\begin{array}{l}\text { Ensayo aleatorizado } \\
\text { por grupos }\end{array}$ \\
\hline $\begin{array}{l}\text { Cell POS (U.Washington, } \\
\text { UPCH, Vía Libre) }{ }^{(10)}\end{array}$ & $\begin{array}{l}\text { Sistemas de } \\
\text { recordatorios para el } \\
\text { paciente }\end{array}$ & $\begin{array}{l}\text { Ensayo controlado } \\
\text { aleatorizado }\end{array}$ \\
\hline $\begin{array}{l}\text { PDA PREVEN } \\
\text { (Universidad Peruana }^{\text {Cayetano Heredia) })^{(11)}}\end{array}$ & Recolección de datos & $\begin{array}{c}\text { Antes y después } \\
\text { (Primera parte) y } \\
\text { Ensayo controlado } \\
\text { aleatorizado } \\
\text { (Segunda parte) }\end{array}$ \\
\hline
\end{tabular}

Uso de PDA

(PNT, Socios en Salud) ${ }^{(12)}$

Uso de PDA

Recolección de datos Encuesta al usuario

\begin{tabular}{|c|c|c|}
\hline (PNT, Socios en Salud) ${ }^{(12)}$ & Recolección de datos & Análisis de costos \\
\hline 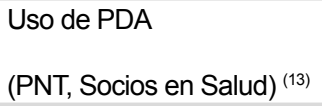 & Recolección de datos & $\begin{array}{l}\text { Ensayo controlado } \\
\text { aleatorizado por } \\
\text { grupos }\end{array}$ \\
\hline $\begin{array}{l}\text { Proyecto Tu Nexo } \\
\left(\text { Vía Libre, UPCH, UW) }{ }^{(14)}\right.\end{array}$ & $\begin{array}{l}\text { Sistemas de } \\
\text { recordatorios para el } \\
\text { paciente }\end{array}$ & $\begin{array}{l}\text { Ensayo controlado } \\
\text { aleatorizado }\end{array}$ \\
\hline
\end{tabular}

Alerta (NMRCD, Peruvian Navy) ${ }^{(15)}$

Recolección de datos Estudio prospectivo

\begin{tabular}{llc}
\hline Cell PREVEN (UPCH) & Recolección de datos & Estudio descriptivo \\
\hline $\begin{array}{l}\text { BK CX Lima Este (Socios } \\
\text { en Salud) }\end{array}$ & Recolección de datos & $\begin{array}{c}\text { Estudio costo- } \\
\text { efectividad }\end{array}$
\end{tabular}

\begin{tabular}{|c|c|c|}
\hline $\begin{array}{l}\text { NETLAB (Instituto } \\
\text { Nacional de Salud) }{ }^{(18)}\end{array}$ & $\begin{array}{c}\text { Sistema de información } \\
\text { de laboratorio }\end{array}$ & Encuestas \\
\hline Wawared (UPCH/BID) ${ }^{(19)}$ & $\begin{array}{l}\text { Sistema de } \\
\text { recordatorios para el } \\
\text { paciente }\end{array}$ & $\begin{array}{l}\text { Ensayo controlado } \\
\text { aleatorizado }\end{array}$ \\
\hline
\end{tabular}

Programa EHAS ${ }^{(20)} \quad \begin{gathered}\text { Sistema de monitoreo, } \\ \text { seguimiento y } \\ \text { evaluación de } \\ \text { pacientes }\end{gathered} \quad$ Costo-efectividad

Sistema de monitoreo, evaluación de pacientes

Programa EHAS (21)

Sistema de monitoreo, seguimiento $y$ evaluación de pacientes

Encuestas

\section{Resultados}

Aumento de la demanda en 39,25\% entre 2010 y 2014

La exactitud de la predicción por medicamento fue $117 \%$ de sobrestimación en 2002 , $5 \%$ de subestimación en 2003 y $2 \%$ de subestimación en 2004.

Para un subgrupo de 58 pacientes en tratamiento individualizado, el modelo predijo $99 \%$ de uso actual, mientras que el orden actual fue $145 \%$ de uso actual. El margen de error disminuyó de $17,4 \%$ a 3,1\% por paciente. El margen de error no difirió significativamente en el grupo control $(8,6$ a $6,9 \%)$.

El grupo de intervención necesitó menos tiempo para recibir los test de susceptibilidad para tuberculosis ( 9 versus 16 días) y para recibir los resultados de cultivo (4 versus 8 días)

El grupo de intervención fue 2,8 veces más adherente que el control (escala SMAQ: Simplified Medication Adherence Questionnaire) y 5,5 veces más adherente usando la escala análoga visual.

El uso de PDA tuvo $96 \%$ menos inconsistencias y $66 \%$ menos datos perdidos que el papel.

La satisfacción de uso fue mayor en los usuarios de PDA versus el papel. Con el uso de PDA se redujo el tiempo de trabajo de 6,7 a 2 minutos.

El número de horas de trabajo disminuyó en $60 \%$. El costo total y el tiempo para desarrollar e implementar fue de 26092 USD y 22 semanas. El costo de expansión en 9 distritos fue 1245 USD y el costo de la implementación fue 4107 USD. Los sistemas que utilizan PDA disminuyen el tiempo de procesamiento de 23 a 8 días y los resultados con tiempo mayor de 90 días, de 9,2 a 0,1\%. Reduce errores en $57,1 \%$.

Los participantes que fueron asignados a la exposición al video, demostraron mayor interés por realizarse la prueba de $\mathrm{VIH}$, aunque la diferencia con el grupo control no fue significativa.

El reporte oportuno se incrementó de 64,6 a $84 \%$ en las clínicas y de 46,9 a $77,3 \%$ en los buques, ambas diferencias significativas, aunque no mejoró la calidad de la información. Se obtuvo 797 reportes, de los cuales 60 (7,5\%) informaban al menos un efecto adverso.

El sistema disminuyo los retrasos en procesamiento y los errores, con experiencia positiva para el usuario. El costo total del sistema fue menos de 600 USD.

El conocimiento del uso del NETLAB por los pacientes se incrementó de 9,5 a 52,3\%.

Los SMS fueron efectivos para incrementar la demanda de control prenatal en grupos con mayor nivel educativo. Los resultados de cambio de conducta y de nacimientos mejoraron solo en el grupo con mayor nivel educativo.

El ahorro neto en cuatro años fue 320126 USD mediante el uso del servicio de telemedicina.

El $90 \%$ del personal de salud percibe que correo electrónico puede reducir las referencias en más de $50 \%$ de los casos.

La media para derivar a un paciente se redujo de 8,6 a $5,2 \mathrm{~h}$. El personal reportó que en el $28 \%$ de los casos de emergencia, la telemedicina salvó la vida del paciente. 
eSalud. Curioso et al. describieron la metodología con la que evaluaron un curso de Bioinformática e Informática para la Salud Pública, tanto los conocimientos y las actitudes adquiridas, antes, durante y después del curso, con resultados muy positivos ${ }^{(23)}$

El Centro Andino de Investigación y Capacitación en Informática Global Salud (QUIPU) evaluó las necesidades de capacitación e investigación en Bioinformática e Informática Médica en profesionales de Perú y Latinoamérica, resaltando la importancia de la evaluación sistemática para mejorar los planes de estudios de estos programas ${ }^{(24)}$.

Otro estudio evaluó el programaAMAUTA, que ofrece cursos cortos sobre Bioinformática e Informática Médica, y describe cómo se realizaron las evaluaciones de conocimientos, antes y después de los cursos, demostrando que, incluso, luego de seis meses, los alumnos mantenían un nivel similar de conocimientos ${ }^{(25)}$. La evaluación de los cursos en eSalud y áreas afines es imprescindible para poder demostrar su eficacia y aceptación, y así poder incrementar sus probabilidades de ser sostenibles en el tiempo. Es posible que, a medida que se incremente el interés y el acceso a las tecnologías de información, más profesionales se interesen por adquirir estos conocimientos para aplicarlos en las diferentes áreas de la salud.

Es importante destacar la relativa escasez de reportes de evaluación de proyectos de eSalud publicados en el Perú. Si bien los datos que se observan muestran resultados positivos, estos no son suficientes, tomando en cuenta el auge de proyectos en eSalud. Además, se observa que de los reportes de evaluación de programas e iniciativas en eSalud, muy pocos fueron ensayos controlados y aleatorizados. Asimismo, un beneficio relacionado a la evaluación de los programas de eSalud es la disminución de los costos de ejecución. Por ejemplo, se encontró una evaluación económica de un proyecto de telemedicina (EHAS-Alto Amazonas), donde se estimó el efecto económico neto sobre un periodo de cuatro años, con una conclusión claramente positiva, cuantificando un ahorro neto anual de USD $320126^{(20)}$.

Idealmente, cada proyecto, independientemente del área que comprenda, debería reportar sus resultados. Existe la posibilidad que no se considere una metodología estricta para la evaluación de proyecto o se utilicen métodos poco específicos. Diversos autores han propuesto estrategias para la evaluación de proyectos de eSalud, las cuales se mencionan a continuación.

\section{ESTRATEGIAS DE EVALUACION MULTIDISCIPLINARIA EN PROGRAMAS E INTERVENCIONES DE SALUD ELECTRÓNICA}

Las evaluaciones de los programas e intervenciones de salud electrónica deben ser planificadas desde la redacción del protocolo, y debe asignarse un presupuesto y tener un cronograma específico para ello. Además, debe ser sistemática y continua para detectar posibles amenazas en el transcurso de la intervención, que son frecuentes en los proyectos que involucran TIC (errores de funcionamiento, baja confiabilidad y problemas relacionados con el usuario).

Es importante resaltar que estas evaluaciones deben realizarse desde un enfoque multidisciplinario, es decir, deben involucrar a diversos profesionales y personal del sector Salud e, incluso, de diferentes sectores, guiados por un grupo de expertos en el área. Una visión desde diferentes perspectivas puede asegurar que se aborden temas culturales, organizacionales, entre otros, que pueden afectar la adaptabilidad de la intervención al usuario final.

A continuación, se presenta una tabla con las principales recomendaciones para la evaluación de intervenciones de eSalud, según autores con amplia experiencia internacional en el área (Tabla 2).

Tabla 2. Preguntas estratégicas a considerar por el evaluador antes de implementar un programa o intervención de salud electrónica

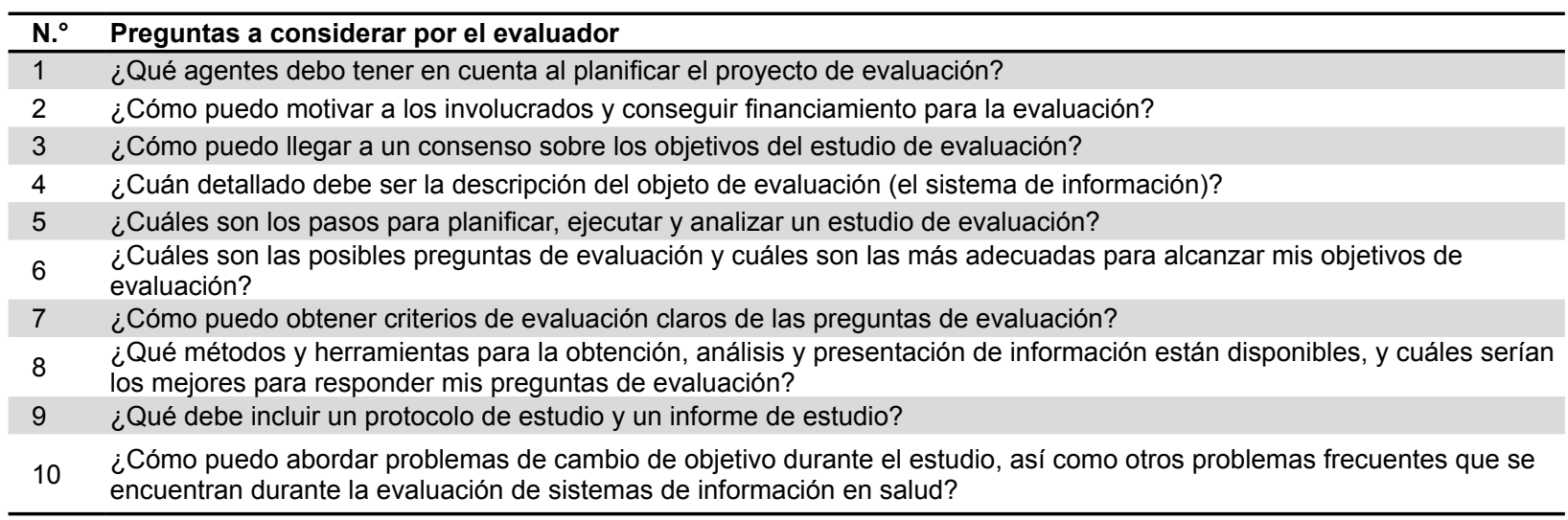




\section{GENERACIÓN DE EVIDENCIA DESDE LA EVALUACIÓN DE INTERVENCIONES EN SALUD ELECTRÓNICA}

La publicación de los resultados de la evaluación de eSalud genera evidencia valiosa para futuros emprendimientos. Existe un creciente número de revistas especializadas en informática de la salud, que proveen plataformas para autores que deseen publicar sus resultados. Existen formatos ya establecidos para la redacción de publicaciones, que han sido adoptados por reconocidas revistas científicas del área, con el fin de mejorar y estandarizar los informes de evaluación. Sin embargo, es importante precisar que no necesariamente una demanda creciente de nuevos programas y proyectos de eSalud, implicará un aumento de las publicaciones en revistas indizadas.

\section{EVALUANDO PROGRAMAS \\ E INICIATIVAS DE SALUD ELECTRÓNICA EN EL PERÚ}

En Perú, el plan de evaluación también debe considerar otras variables. Para asegurar el éxito de las intervenciones de eSalud se debe tomar en cuenta la infraestructura y los recursos tecnológicos que son necesarios para asegurar la correcta implementación y sostenibilidad en el tiempo. El acceso a la telefonía y a internet es aún limitado, sobre todo en áreas remotas del Perú (28). Sin embargo, la apertura a nuevas compañías de telefonía móvil podría generar un escenario favorable para el incremento de cobertura, en especial en la Amazonía. Es muy importante considerar la percepción del usuario final respecto al uso de las TIC en reemplazo de métodos tradicionales, como los registros escritos, por la posibilidad de dificultades en el proceso de transición ${ }^{(15)}$

\section{CONCLUSIONES}

El creciente acceso a las TIC genera mayor interés en desarrollar nuevas iniciativas de eSalud, con el fin de superar las brechas de acceso en la población. La evaluación rigurosa es de gran importancia para los tomadores de decisiones en gestión en salud, y para mejorar la calidad de los servicios que se brindan a los usuarios, también es necesario considerar el entorno y el ecosistema en el que se utilizan las TIC en salud. Nuestra búsqueda permitió identificar un número relativamente bajo de publicaciones relacionadas a la evaluación de programas e iniciativas de eSalud en Perú, comparado con el número de proyectos implementados durante el periodo explorado.

Sin embargo, para poder mantener los proyectos en marcha, no solo se debe concentrar esfuerzos en la implementación, sino se debe mantener un plan de evaluación continua que involucre a equipos multidisciplinarios, con indicadores preestablecidos que permitan, entre otros factores, detectar riesgos que puedan comprometer el éxito de estas innovaciones. Es necesario la inclusión de un plan detallado de evaluación en los proyectos y protocolos de investigación, para asegurar un adecuado monitoreo de las intervenciones, así como buscar oportunamente fuentes de financiamiento para lograrlo.

Hoy en día existen mecanismos de financiamiento a nivel nacional para proyectos TIC promovidos por el Consejo Nacional de Ciencia, Tecnología e Innovación Tecnológica (CONCYTEC) a través de las convocatorias de Cienciactiva (www.cienciactiva.gob.pe/) y las oportunidades del Ministerio de la Producción, a través del Programa Nacional de Innovación para la Competitividad y Productividad (Innóvate Perú) (www.innovateperu.gob.pe/). Asimismo, a pesar de que las evaluaciones de los proyectos y programas de eSalud identificados muestran un impacto positivo e, incluso, costo efectivo, todavía muchos de los proyectos y programas no han sido escalados, ni implementados a gran medida a nivel del Estado.

Algunos factores que se pueden mencionar, relacionados con la falta de incorporación de los proyectos y programas de salud electrónica al sistema de salud y que también han sido reportadosen otros países ${ }^{(29,30)}$, incluyen: falta de conocimiento de los tomadores de decisión del impacto positivo de los proyectos y programas de salud electrónica a nivel global y local; resistencia al cambio de los profesionales de salud para la adopción de los proyectos y programas de eSalud en sus instituciones; falta de medios y mecanismos apropiados para la difusión de las evaluaciones de los proyectos y programas de eSalud; voluntad política para colocar la telesalud y, en general, la eSalud como tema prioritario para lograr eficiencia y fomentar la transparencia en el sistema de salud; falta de incentivos para el personal sanitario para el uso de la telesalud y registros electrónicos; falta de infraestructura tecnológica apropiada; gran diversidad de registros y aplicaciones electrónicas dispersas y no conectadas unas con otras; complejidad en el uso de las soluciones implementadas; cuestiones de seguridad, privacidad y confidencialidad de la información de las personas; alta rotación del personal sanitario; falta de alineación estratégica entre los distintos participantes; falta de apropiación, sensación de pertenencia y vínculo con el proyecto de salud electrónica; la alta carga de trabajo del personal de salud; distintos intereses; preocupaciones y prioridades del personal de salud para poner en práctica los servicios relacionados a la eSalud y los costos de implementación.

Finalmente, se debe sistematizar la información existente, generando observatorios de evaluación de eSalud en el Perú, a través de los repositorios o portales, donde se compartan buenas prácticas de gestión, similar a lo propuesto en la región ${ }^{(1)}$. Esta valiosa información logrará que futuros programas sean de mejor calidad, respondan a las prioridades de salud del país y permitan potenciar la colaboración internacional. 
Contribuciones de los autores: MHS, CEBV, ELLF y EEP participaron en la concepción, diseño y redacción del manuscrito de manera conjunta. Todos los autores aprobaron la versión final a publicar, y asumen la responsabilidad frente a todos los aspectos del manuscrito.
Fuente de financiamiento: autofinanciado.

Conflictos de interés: los autores declaran no tener ningún conflicto de interés.

\section{REFERENCIAS BIBLIOGRÁFICAS}

1. Organización Mundial de la Salud. 58th World Health Assembly Report, resolutions and decisions [Internet]. Ginebra: OMS; 2005 [citado el 5 de junio de 2017]. Disponible en: http:// www.who.int/healthacademy/media/ WHA58-28-en.pdf

2. Organización Panamericana de la Salud. Estrategia y Plan de Acción sobre eSalud (2012 - 2017). Washington DC: OPS/ PAHO; 2011. [citado el 5 de junio de 2017]. Disponible en: http://www.paho. org/ict4health/index.php?option=com content\&view $=$ article\&id $=54 \% 3$ Aestrat egia-y-plan-de-accion-sobre-esalud-20122017\&Itemid $=146 \&$ lang $=\mathrm{es}$

3. Gozzer Infante E. Una visión panorámica de las experiencias de Telesalud en Perú. Rev Peru Med Exp Salud Publica. 2015;32(2):385-90.

4. Blaya JA, Fraser HSF, Holt B. E-health technologies show promise in developing countries. Health Aff (Millwood). 2010;29(2):244-51. doi: 10.1377/hlthaff.2009.0894.

5. Vargas-Herrera J, Segovia-Juarez J, Garro Nuñez GM. Sistema de información de la red nacional de laboratorios de salud pública en el Perú (Netlab). Rev Peru Med Exp Salud Publica.2015 ;32(2):378-84.

6. Fraser H, Jazayeri D, Choi S, Blaya JA, Bayona J, Levison L, et al. Forecasting three years drug supply for a large MDRTB treatment program in Peru. Int J Tuber Lung Dis. 2006;10(11 Supp 1):S245.

7. Yamanija J, Durand R, Bayona J, Blaya JA, Jazayeri D. Comparing actual medication consumption against the quantities ordered and a prediction using an information system. Int J Tuber Lung Dis. 2006;10(11 Supp 1):S69-70.

8. Choi SS, Jazayeri DG, Mitnick CD, Chalco K, Bayona J, Fraser HS. Implementation and initial evaluation of a Web-based nurse order entry system for multidrug-resistant tuberculosis patients in Peru. Stud Health Technol Inform. 2004;107(Pt 1):202-6.
9. Blaya JA, Shin S, Contreras C, Yale G, Suarez C, Asencios L, et al. Full impact of laboratory information system requires direct use by clinical staff: cluster randomized controlled trial. J Am Med Inform Assoc. 2011;18(1):11-6. doi: 10.1136/jamia.2010.005280.

10. Curioso WH. Evaluation of a Computer-Based System using Cell Phones for HIV positive people in Peru [tesis doctoral]. [Seattle]: Universidad de Washington; 2012. [citado el 20 de marzo de 2017]. Disponible en: https://digital. lib.washington.edu/researchworks/bitstream/handle/1773/20614/Curioso_ washington_0250E_10684.pdf?sequen$\mathrm{ce}=1$ \&isAllowed $=\mathrm{y}$

11. Bernabe-Ortiz A, Curioso WH, Gonzales MA, Evangelista W, Castagnetto JM, Carcamo CP, et al. Handheld computers for self-administered sensitive data collection: A comparative study in Peru. BMC Med Inform Decis Mak. 2008;8(11). doi: 10.1186/1472-6947-8-11.

12. Blaya JA, Gomez W, Rodriguez P, Fraser H. Cost and implementation analysis of a personal digital assistant system for laboratory data collection. Int $\mathrm{J}$ Tuberc Lung Dis. 2008;12(8):921-7.

13. Blaya JA, Cohen T, Rodríguez P, Kim J, Fraser HSF. Personal digital assistants to collect tuberculosis bacteriology data in Peru reduce delays, errors, and workload, and are acceptable to users: cluster randomized controlled trial. Int J Infect Dis. 2009;13(3):410-8.

14. Blas MM, Alva IE, Carcamo CP, Cabello R, Goodreau SM, Kimball AM, et al. Effect of an Online Video-Based Intervention to Increase HIV Testing in Men Who Have Sex with Men in Peru. PLoS One. 2010;5(5):e10448. doi: 10.1371/journal.pone.0010448.

15. Huaman MA, Araujo-Castillo RV, Soto G, Neyra JM, Quispe JA, Fernandez MF, et al. Impact of two interventions on timeliness and data quality of an electronic disease surveillance system in a resource limited setting (Peru): a prospective evaluation. BMC Med Inform Decis Mak. 2009;10;9:16. doi: 10.1186/1472-6947-9-16.

16. Curioso WH, Karras BT, Campos PE, Buendia C, Holmes KK, Kimball AM. Design and implementation of CellPREVEN: a real-time surveillance system for adverse events using cell phones in Peru. AMIA Annu Symp Proc. 2005: 176-80.

17. Blaya J, Fraser HS. Development, implementation and preliminary study of a PDA-based tuberculosis result collection system. AMIA Annu Symp Proc. 2006:41-5.

18. García PJ, Vargas JH, Caballero NP, Calle VJ, Bayer AM. An e-health driven laboratory information system to support HIV treatment in Peru: E-quity for laboratory personnel, health providers and people living with HIV. BMC Med Inform Decis Mak. 2009;9:50. doi: 10.1186/1472-6947-9-50.

19. Beuermann DW, Anta R, Curioso WH, Garcia PJ, Maffioli A, Pérez-Lu JE, et al. Determinants and effects of prenatal care services: evidence from a mobile phone RCT [Internet]. Social Protection and Health Division; 2017. [citado el 15 de junio de 2017]. Disponible en: https:// issuu.com/bid-sph/docs/spd_04_17_14

20. Martínez A, Villarroel V, Puig-Junoy J, Seoane J, del Pozo F. An economic analysis of the EHAS telemedicine system in Alto Amazonas. J Telemed Telecare. 2007;13(1):7-14. doi: $10.1258 / 135763307779701176$

21. Martínez A, Villarroel V, Seoane J, del Pozo F. Analysis of information and communication needs in rural primary health care in developing countries. IEEE Trans Inf Technol Biomed. 2005;9(1):66-72.

22. Martínez A, Villarroel V, Seoane J, del Pozo F. A study of a rural telemedicine system in the Amazon region of Peru. 
J Telemed Telecare. 2004;10(4):219-25. doi: 10.1258/1357633041424412.

23. Curioso WH, Hansen JR, CenturionLara A, Garcia PJ, WolfFM, Fuller S, et al. Evaluation of a joint Bioinformatics and Medical Informatics international course in Peru. BMC Med Educ. 2008;8:1. doi: 10.1186/1472-6920-8-1.

24. Blas MM, Curioso WH, Garcia PJ, Zimic M, Carcamo CP, Castagnetto $\mathrm{JM}$, et al. Training the biomedical informatics workforce in Latin America: results of a needs assessment. BMJ Open. 2011 ;1(2):e000233-e000233.

25. Curioso WH, Fuller S, Garcia PJ, Holmes KK, Kimball AM. Ten years of international collaboration in biomedical informatics and beyond: the AMAUTA program in Peru. J Am Med Inform
Assoc. 2010;17(4):477-80. doi: 10.1136/ jamia.2009.002196.

26. Ammenwerth E, Gräber $S$, Herrmann G, Bürkle T, König J. Evaluation of health information systems-problems and challenges. Int J Med Inf. 2003;71(23):125-35.

27. van Gemert-Pijnen JE, Nijland N, van Limburg M, Ossebaard HC, Kelders SM, Eysenbach G, et al. A Holistic Framework to Improve the Uptake and Impact of eHealth Technologies. J Med Internet Res. 2011;13(4):e111. doi: 10.2196/ jmir.1672.

28. Curioso WH, Espinoza-Portilla E. Marco conceptual para el fortalecimiento de los Sistemas de Información en Salud en el Perú. Rev Peru Med Exp Salud Publica. 2015;32(2):335-42.
29. Tracy J, Rheuban K, Waters RJ, DeVany M, Whitten P. Critical steps to scaling telehealth for national reform. Telemed J E Health. 2008;14(9):990-4.

30. Lorenzi NM, Riley RT. Organizational issues = change. Int $\mathrm{J}$ Med Inform. 2003;69(2-3):197-203.

Correspondencia: Elizabeth Espinoza-Portilla Dirección: Instituto de Gobierno y de Gestión Pública. Universidad de San Martín de Porres. Calle Martín Dulanto 101, Urb. San Antonio, Miraflores. Lima, Perú.

Teléfono: (+51) 998796750.

Correo electrónico: espinoza.portilla@gmail.com

\section{Ahora nuestra revista incluye:}

$\checkmark$ Publicación anticipada

Compartiendo publicaciones científicas con el ciudadano

$\checkmark$ Videos de presentaciones conjuntas del Instituto Nacional de Salud y la Academia Nacional de Medicina

$\checkmark$ Galería fotográfica y uideos

www.rpmesp.ins.gob.pe 